\title{
CEA Saclay Codes Review for High Intensities Linacs Computations
}

\author{
Romuald Duperrier, Nicolas Pichoff, Didier Uriot \\ CEA, 91191 Gif sur Yvette Cedex, France \\ rduperrier@cea.fr; npichoff@cea.fr; duriot@cea.fr \\ http://www.cea.fr
}

\begin{abstract}
More and more, computations are playing an important role in the theory and design of high intensities linacs. In this context, CEA Saclay is involved in several high power particles accelerators projects (SNS, ESS, IFMIF, CONCERT, EURISOL) and high intensities protons front end demonstrators (LEDA, IPHI). During these last years, several codes have been developed. This paper consists in a review of several of these codes especially: the linac generator able to design linac structures (computations coupled with SUPERFISH), the TOUTATIS code computing transport in RFQs in 3D grids (multigrid Poisson solver), the TraceWin code allowing to compute end to end simulation of a whole linac (automatic matching, up to 2 millions macroparticles run for halo studies with its PARTRAN module with 3D space charge routines, an errors studies module allowing large scale computations on several PCs using a multiparameters scheme based on a client/server architecture).
\end{abstract}

\section{Introduction}

A high power linacs can only work with very low structure activation. To design the accelerator, a very good estimation of losses location and emittances growth is necessary. In this goal, several transport codes have been developped at CEA Saclay. A lot of work has been performed in order to take into account several effects as space charge (3D routines), image forces, diffusion on the residual gas where it seems to be relevant. Applying basic matching rules, codes for linac generation has also been written and produce robust design. This paper is a review of these different tools.

\section{Linac generation}

\subsection{Basic rules}

For a transport with no emittance growth, a space charge driven beam has to be in equilibrium with the external focusing forces. When this external conditions are changed too abruptly (i.e. adiabatically), the beam reorganizes itself toward 
a new equilibrium. This induces an amount of entropy (emittance growth). Moreover, when transition are smooth, the design is current independant (see figure 1). Linacs generator have been written taking into account such rules for each part of the linac. The following section gives an example with the DTL/SDTL generator.
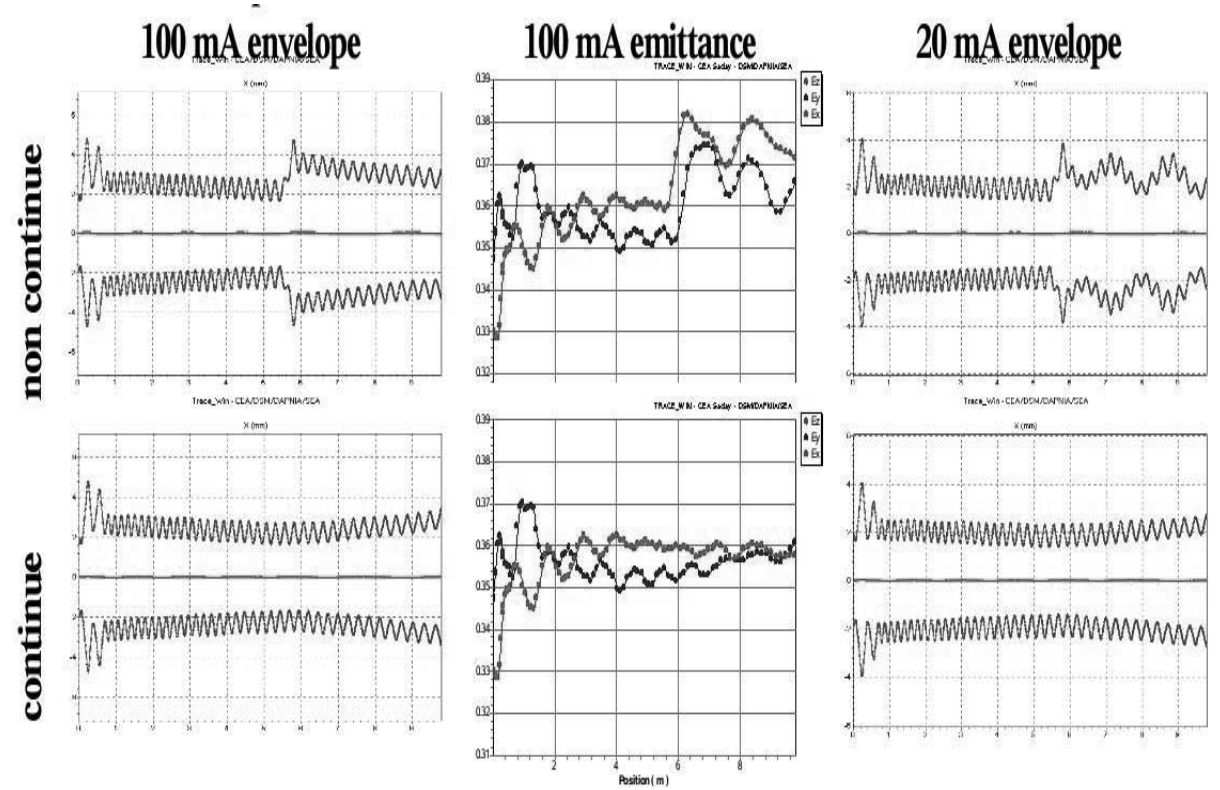

Fig. 1. Plot showing how emittance is affected by discontinuities of phase advance per meter and the current independance of a continuous channel.

\subsection{Example of codes applying these rules}

GenDTL is the DTL and SDTL generator [1]. This code generates a complete design. It may be compared to a pre and post processor for the code SUPERFISH [2]. This radio frequency solver is only used to mesh a particular design and to compute electrical field map. The transport of a synchronous particle through these field map accurately determine phase shift, synchronous phase laws and energy gain. No interpolation is necessary, each cell is computed. The figure 2 shows a snapshot of the generator front end. Applying the rules described above, DTL design without emittance blow up can be easily produced. 

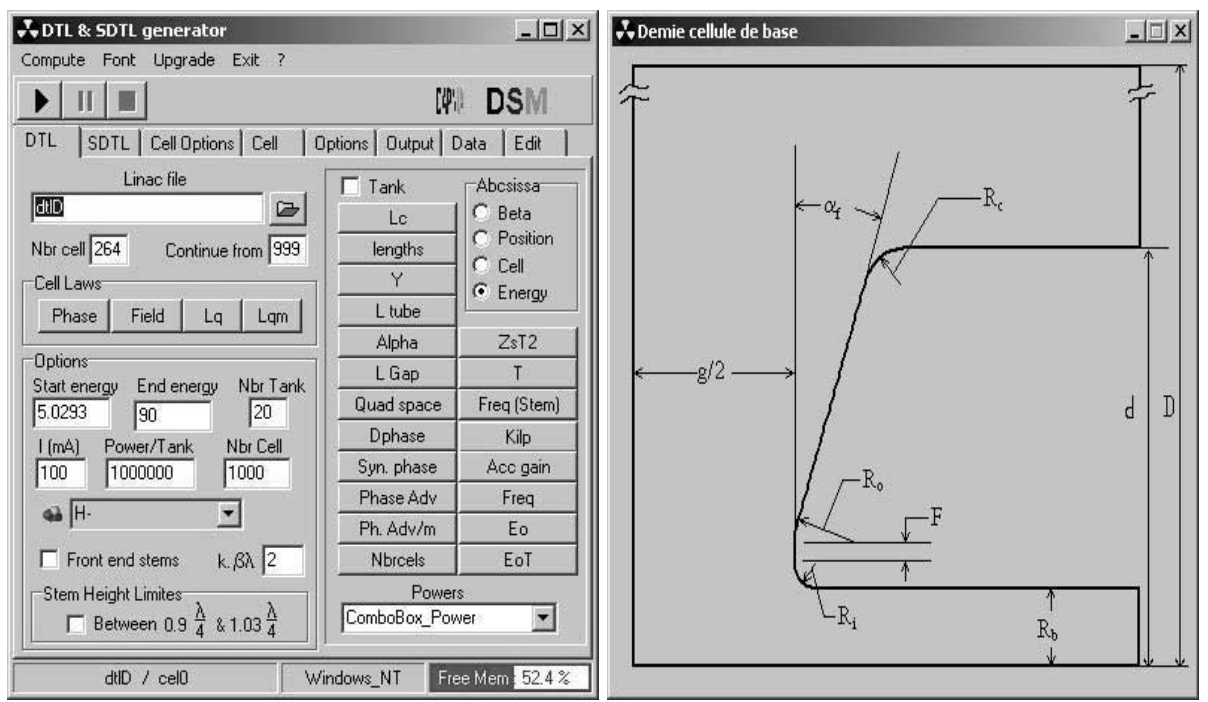

Fig. 2. Snapshot of the generator front end with a drift tube drawing.

\section{TraceWin}

This code is used for linear calculations with 2D and 3D beams [3]. It allows fast calculations. Its input file is written in a language describing linac element and beam characteristics (see figure 3). The current version is running only on win32 platform but have been successfully emulated on a linux machine. TraceWin is able to matched the beam in the whole linac exept the longitudinal plane for bunching RFQ. Either the beam is matched to a channel with lattices (figure 4 ), or gradient, field amplitude in cavity and element length are computed to obtain required beam parameters. Multiparticles codes can be run to validate the linear matching (TOUTATIS, PARTRAN, SCDYN, MONET). The criteria for matching is based on a smooth evolution of phase advance per meter.

The code can plot several results computed by multiparticle codes concerning the linac (lattice length, quads gradients, cavity fields, phase and power) and the beam (emittance, halo parameter, envelops, tune depression, energy and phasespace distributions). The figure 5 gives an example of such plot.

\section{Multiparticles Codes}

\subsection{TOUTATIS}

At low energy, the radio frequency quadrupole (RFQ) is an accelerator element that is very sensitive to losses (sparking). To simulate this structure, a high accuracy in field representation is required because the beam/aperture ratio is often very close to one. TOUTATIS aims to cross-check results and to obtain more 


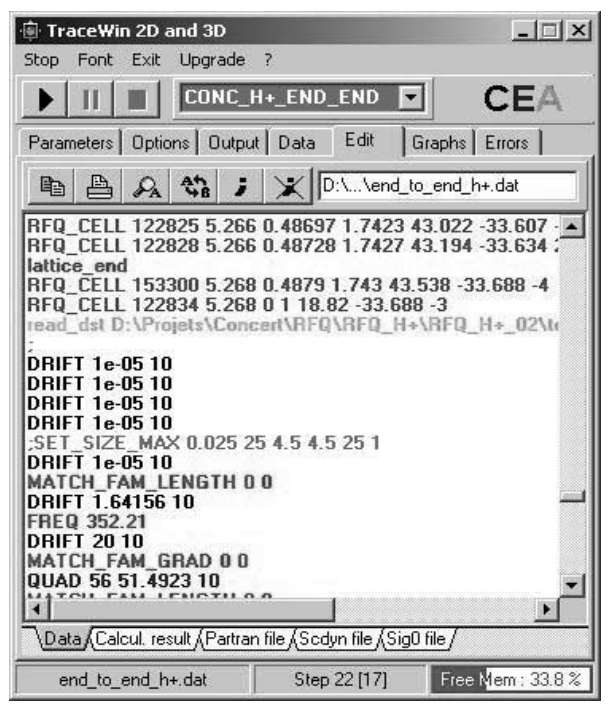

Fig. 3. Snapshot of the editor tabset of TraceWin showing an example for linac description.
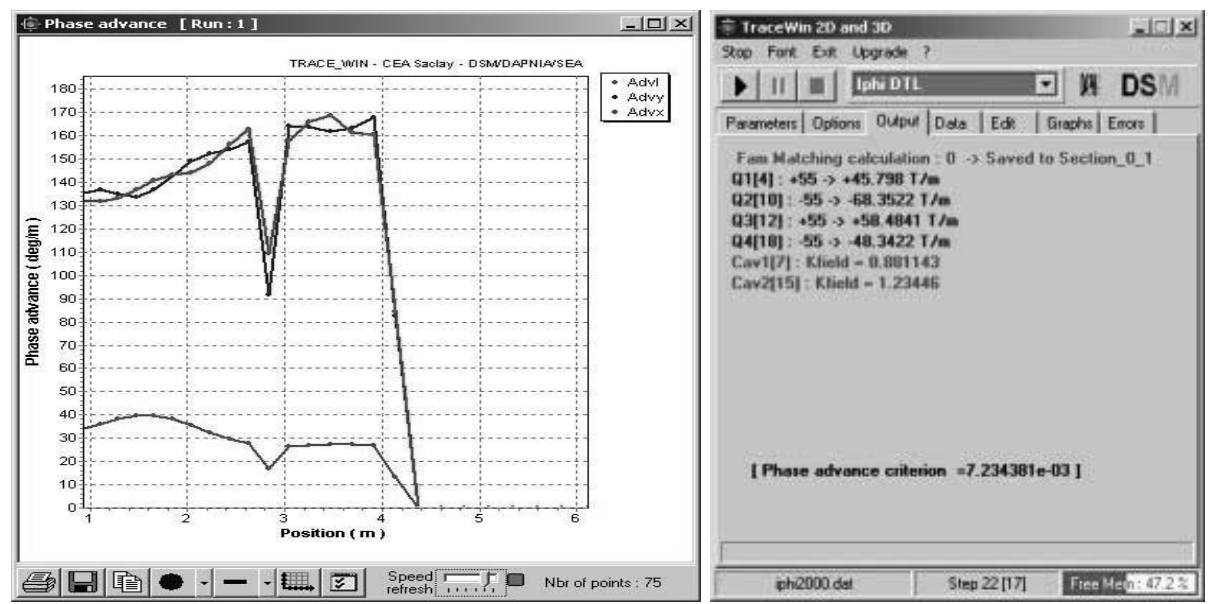

Fig. 4. The output tabset of TraceWin with phase advance per meter plot during a matching procedure.

reliable dynamics. Motion is solved with a symplectic integrator using time as independant parameter. The field is calculated through a Poisson solver and the vanes are fully described. The solver is accelerated by multigrids method (Fig. 6 ). An adaptive mesh for a fine description of the forces is included to compute accurately without being time consuming. This scheme allows to properly simulate the coupling gaps and the RFQs extremities. Theoretical and experimental 

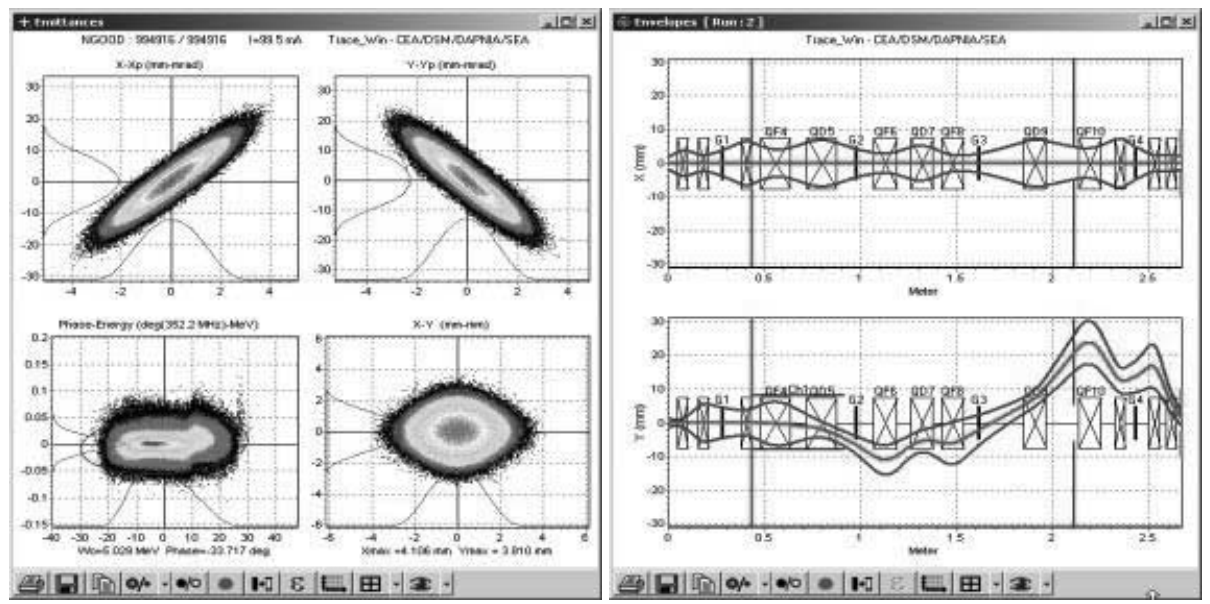

Fig. 5. An example of phase-space distribution plot and envelop evolution in a chopper line.

tests were carried out and showed a good agreement between simulations and reference cases [4].

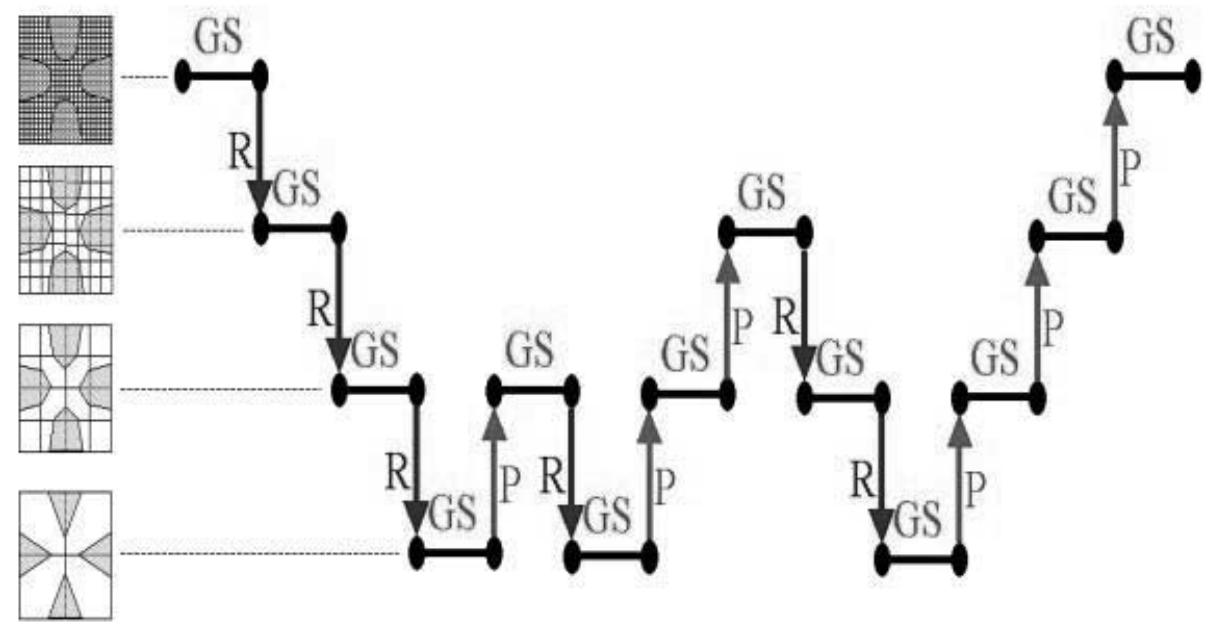

Fig. 6. Representation of the TOUTATIS cycle (GS = 3 Gauss-Seidel, $\mathrm{R}=$ Restriction, $\mathrm{P}=$ Prolongation). 


\subsection{PARTRAN}

PARTRAN (PARticles TRANsport) can simulate the rest of the linac. Motion is calculated with a leap frog. A sophisticated model "sine-like" is included for superconducting cavity, external magnetic field are linear. Space charge effects may be compute using $2 \mathrm{D}$ or $3 \mathrm{D}$ routines (respectively with SCHEFF and PICNIC)[5]. Additional effects as diffusion on the residual gas and stripping can be added. Two stripping phenomena are taken into account:

- interaction with magnetostatic field

- interaction with residual gas

PATRAN is launched using the TraceWin interface as shown by figure 7 . Several elements are available as chopper or funnel cavity. It simulates many linac errors (see following section).

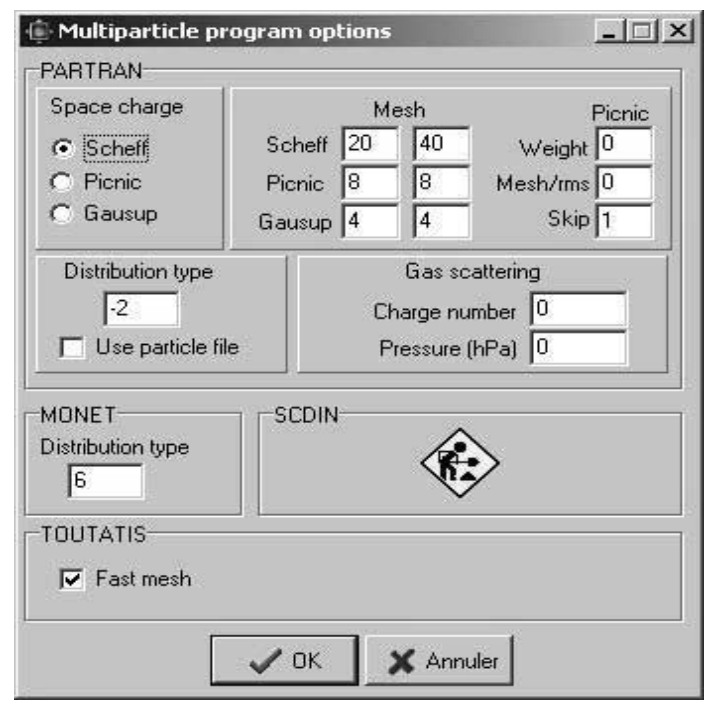

Fig. 7. Configuration window for multiparticle run.

\section{ERRORS STUDIES}

In order to define the tolerances for the linac, errors studies are necessary. These errors are classified as follow:

- Beam errors: displacements, emittance growth, mismatches, current.

- Statistics errors including:

- Quadrupole errors: displacement and rotation in three directions, gradient, combination of errors. 
- Cavity errors: displacement and rotation in two directions, field amplitude, field phase, combination of errors.

- Combination of quad and cavities errors.

Statistics errors may be static, dynamic or both. Dynamic means that correctors are included in the scheme.
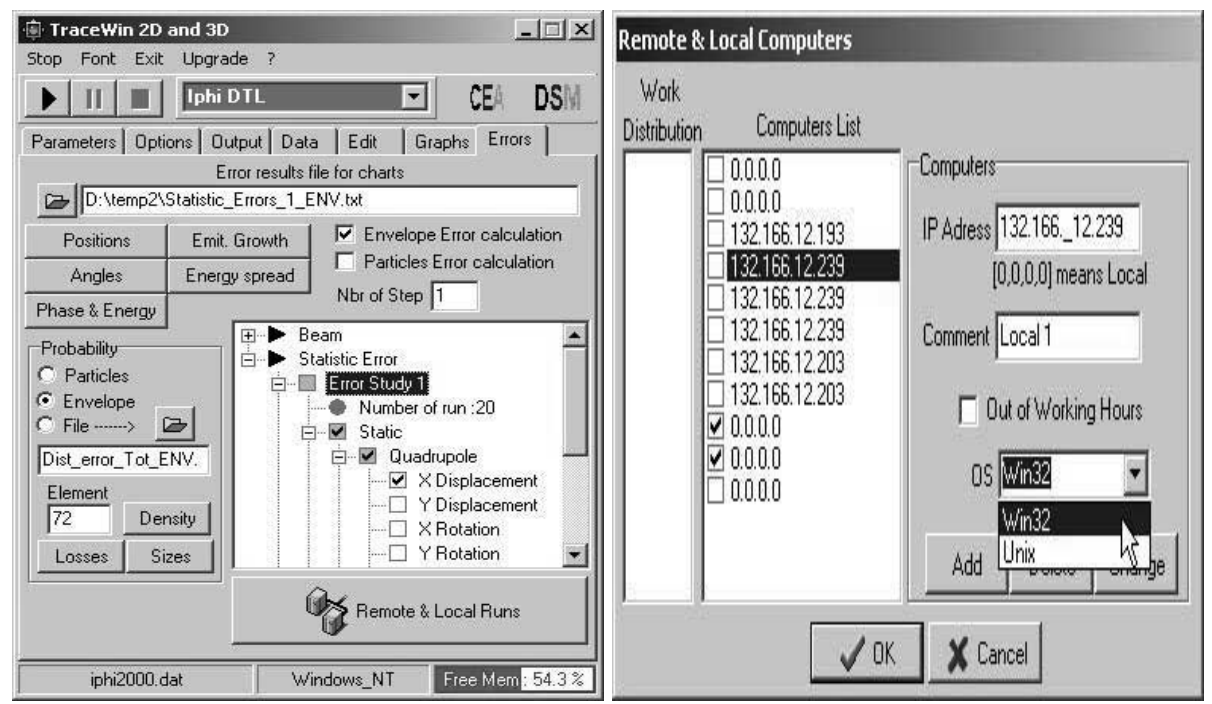

Fig. 8. Snapshot of the Errors tabset and remote runs window of TraceWin.

The TraceWin code is used as interface to manage this part (fig. 8). All these computations are distributed on a heterogen park of machines using a multiparameters scheme based on a client/server architecture. The client and the server are written in Tcl language. This allows to calculate on several platforms (win32, unix, mac) if executables to distribute are available. At present time, only TOUTATIS is multiplatorm but developments are in progress to port PARTRAN and TraceWin on unix platform.

\section{Conclusion and prospects}

Several tools have been developed at CEA Saclay for large scale computations on high power linac during this last years. Every parts of the linac can be simulated. Linac generator produces robust design with very low emittance growth and losses rate. Netherveless, it is still work to do. Space charge compensation can not be predicted at present time by these codes. The package is not completely multiplatform. 


\section{References}

1. N. Pichoff, D. Uriot,"GenDTL", CEA internal report CEA/DSM/DAPNIA/SEA/2000/46 (2000).

2. J. Billen, L. Young, "Poisson Superfish", Los Alamos internal report LA-UR-96-1834 (1996).

3. N. Pichoff, D. Uriot,"TraceWin, documentation", internal report CEA/DSM/DAPNIA/SEA/2000/45 (2000).

4. R. Duperrier,"TOUTATIS: A radio frequency quadrupole code", Phys. Rev. Spec. Topics Acc. \& Beams, Volume 3 (2000).

5. N. Pichoff et al.,"Simulation results with an alternate 3D space charge routine, PICNIC", MO4042, LINAC 1998 conference, Chicago. 